\title{
Evaluation of Changes in Condylar Cartilage Thickness Using MRI and Ultrasound Imaging in Patients Treated by Mandibular Advancement With Myofunctional Appliance: An In-Vivo Pilot Study
}

\author{
Sonali Deshmukh ${ }^{1}$, Sachin Durkar ${ }^{2}$, Amit Kharat ${ }^{3}$, Suramya Shiranjani ${ }^{4}$, Pranav Ajmera ${ }^{5}$ \\ 1. Orthodontics and Dentofacial Orthopaedics, Dr. D.Y. Patil Dental College, Dr. D.Y. Patil Vidyapeeth (DPU), Pune, IND \\ 2. Orthodontics, Dr. D.Y. Patil Dental College, Dr. D.Y. Patil Vidyapeeth (DPU), Pune, IND 3. Musculoskeletal Radiology, \\ Dr. D.Y. Patil Medical College, Hospital and Research Center, Dr. D.Y. Patil Vidyapeeth (DPU), Pune, IND 4. Dentistry, \\ Dr. D.Y. Patil Dental College, Dr. D.Y. Patil Vidyapeeth (DPU), Pune, IND 5. Radiology, Dr. D.Y. Patil Medical College, \\ Hospital and Research Center, Dr. D.Y. Patil Vidyapeeth (DPU), Pune, IND
}

Corresponding author: Pranav Ajmera, pranavajmera@gmail.com

\section{Abstract}

\section{Introduction}

The changes occurring due to growth modulation of the condylar cartilage act as an important mechanism for mandibular advancement using myofunctional appliance therapy. So this study aims to evaluate the condylar cartilage thickness by using MRI and USG in patients undergoing myofunctional appliance therapy for mandibular advancement with the null hypothesis being that there are no changes seen in the thickness of condylar cartilage in growing children.

\section{Materials and methods}

A prospective evaluation of samples having skeletal Class-II malocclusion ranging between cervical vertebral maturation index (CVMI) stage II and III, requiring twin block functional therapy was performed. Ten patients were selected randomly who underwent MRI and USG in the open and close positions for the evaluation of condylar cartilage thickness and the dimensional changes in the width of the right and left condyle in $\mathrm{mm}$ at $\mathrm{T} 0$ and $\mathrm{T} 1$.

\section{Result}

There was no statistically significant difference between the values interpreted by MRI or USG imaging when compared at $\mathrm{T} 0$ and $\mathrm{T} 1$ and in the open and closed mouth on the left and right sides. At T0, the mean thickness noted was $0.49 \mathrm{~mm}$ and $0.48 \mathrm{~mm}$ during opening and closing on the left side and $0.52 \mathrm{~mm}$ in both positions on the right side. At T1, the mean thickness noted was 0.8 and $0.79 \mathrm{~mm}$ during opening and closing

Review began 06/20/2021 Review ended 06/30/2021 Published 07/12/2021

() Copyright 2021

Deshmukh et al. This is an open access article distributed under the terms of the Creative Commons Attribution License CC-BY 4.0., which permits unrestricted use, distribution, and reproduction in any medium, provided the original author and source are credited. on the left side, whereas it was $0.81 \mathrm{~mm}$ in both positions on the right side.

\section{Conclusion}

The condylar cartilage thickness increases significantly after twin block therapy suggestive of mandibular growth in skeletal class II malocclusion. It can be inferred that both MRI and USG carry equal diagnostic interpretation, as there was no statistically significant difference between the two imaging modalities.

Categories: Otolaryngology, Radiology, Other

Keywords: condylar cartilage, mri, usg, myofunctional appliance therapy, functional therapy

\section{Introduction}

The prevalence of class-II malocclusion is about $8.37 \%$ in the Indian population. Class-II division 1 malocclusion is often associated with discrepancies like narrow maxilla, high arched palate, increased overjet, and microgenia. However, the most commonly associated feature is mandibular skeletal retrusion. Functional appliance therapy serves to enhance mandibular growth, thereby correcting the skeletal discrepancy [1].

Most of the functional appliances are designed for propagating forward growth of the mandible and inducing displacement of the mandibular condyles in a downward and forward direction. Adaptive remodeling occurs on both the articular surfaces of the temporomandibular joint, thrusting the mandible forward and improving the sagittal position of the mandible relative to the maxilla [1]. 
Mandibular advancement induced by functional therapy for skeletal Class-II malocclusion is a principal factor that directs cellular activities during tissue morphogenesis and mechanical stress. Forward positioning of the mandible is a primary event that is followed by adaptive remodeling in the mandibular condylar cartilage (MCC) and the glenoid fossa (GF). Bone remodeling occurs by the expression of endogenous regulatory factors of cells in the mandibular condyle through an endochondral and intramembranous ossification in the GF [2].

As conventional imaging systems acquire several limitations, a detailed study of the temporomandibular joint (TMJ) structure using magnetic resonance imaging (MRI) and ultrasound imaging is substantiated since these imaging modalities offer a method for diagnostic superiority for the evaluation of both the soft and hard tissues of the TMJ. MRI facilitates the non-invasive assessment of various tissues by providing highresolution images that account for apparent differentiation among soft tissues. The clinical application of MRI has prompted a better understanding of the anatomy, growth, and disease of several joints of the human body, including the temporomandibular joint [3].

Animal studies have suggested that there is temporal bone and condylar adaptation in response to protrusive mandibular function. Shen and Darendeliler documented that the adaptive remodeling of the condylar cartilage proceeds with the biomolecular pathway initiating from chondrogenesis followed by osteogenesis. The theory of growth relativity essentially states that bone growth modification occurs relative to two elements: the retrodiscal tissues are stretched reciprocally, similar to a large elastic band, between the fossa and the displaced condyle during the expansion of the growing facial complex; and the transduction of these non-muscular forces has been shown to be effective at a significant distance from the actual physical soft tissue attachments [4].

Various techniques have been used to view the TMJ prior to the advent of MRI, which include lateral cephalograms, orthopantomograms and tomograms, bone scintigraphy with radiologic markers like $99 \mathrm{mTc}-$ MDP, arthroscopy, arthrography, and CT scanning. MRI, which is essentially a multiplanar imaging technique, is advantageous, owing to its ability to provide an accurate assessment of bony and soft tissues. Currently, MRI is opted for as the imaging modality of choice for assessing internal derangements of the temporomandibular joint. Ultrasonography (USG) is a highly reproducible method to evaluate the mandibular condyle cartilage, which is an economical and cost-effective diagnostic imaging method and could help study changes in the condylar cartilages [5].

On thorough examination of the currently available literature, functional changes, including bone remodeling and joint adaptations, have been studied using X-rays, histologic studies, electromyographic studies (EMG), ultrasound imaging, MRI, and computed tomography. In a study by TG Williams et al., bone appearance and shape were used to assess changes in cartilage thickness over time for individuals suffering from osteoarthritis (OA) [6].

Schmitz RJ et al. undertook a study to better understand how well clinical ultrasound thickness measures are associated with gold standard MRI measures by analyzing the femoral condylar cartilage thickness in OA patients. They concluded that there was a moderate to strong correlation between the MRI and USG measure of cartilage thickness in the medial femoral condyle [7].

However, no studies quantified the condylar cartilage activity in humans using MRI and USG. There is a need to develop easily accessible and cost-effective clinical tools to assess the changes in articular cartilage thickness.

Thus, the aim of this study is to evaluate the condylar cartilage thickness by using MRI and ultrasound imaging in patients undergoing myofunctional appliance therapy for mandibular advancement and to compare between MRI and USG.

\section{Materials And Methods}

A prospective evaluation involving convenience sampling of 10 participants was undertaken. The sample size was decided by using the formula:

$\mathrm{n}=\mathrm{N} * \mathrm{X} /(\mathrm{X}+\mathrm{N}-1)$, where $\mathrm{X}=\mathrm{Z} \alpha / 22 * \mathrm{p} *(1-\mathrm{p}) /(\mathrm{MOE}) 2$

The inclusion criteria involved growing patients exhibiting skeletal Class-II malocclusion with retrognathic mandible, patients exhibiting growth status in between CVMI II and III, patients undergoing myofunctional appliance therapy using a twin-block appliance, patients exhibiting a normodivergent to hypodivergent growth pattern. The exclusion criteria involved non-growing patients and patients exhibiting a growth status of CVMI IV. The diagnosis of participants who were given a twin block was based on clinical and radiological findings. The duration of the study was 18 months. For all patients, construction bite with horizontal advancement ranging between $5 \mathrm{~mm}$ and $6 \mathrm{~mm}$ and vertical advancement within freeway space ranging between $3 \mathrm{~mm}$ and $4 \mathrm{~mm}$ was obtained. All patients underwent MRI and ultrasound imaging to evaluate condylar cartilage thickness. The dimensional changes of the width of the condyle at the beginning 


\section{Cureus}

(T0) and after six months of twin-block therapy (T1) were calculated by a single examiner who was a highly trained radiologist. Intraobserver reliability was 0.93 . The thickness of condylar cartilage was measured in the area where maximum proliferative changes were seen. The USG and MRI were taken at some point of time but the operator was blinded for the measurement on MRI since it was measured at a different point of time, whereas the operator performed USG measurements chairside at the same point of time; this was done to avoid bias between USG and MRI readings. The final treatment results were not included in the study, as we wanted to primarily assess the changes in condylar cartilage thickness using different imaging modalities.

The MRI scanner used was the Siemens 1.5 Tesla (Siemens AG, Munich, Germany) utilizing both the surface and brain coils. As part of the protocol, thin coronal and sagittal sections of the temporomandibular joint were acquired in both the closed and open mouth positions in two separate sessions labeled T0 and T1. The ultrasound scanner used for the study was the Hitachi-Aloka system (ProSound Alpha-6; Hitachi, Ltd.,

Tokyo, Japan) with a linear transducer working within the 7-12Mhz frequency bandwidth. Patient position for MRI and ultrasound imaging is illustrated in Figure 1. The sagittal view of the temporomandibular joint using the MRI imaging modality is shown in Figure 2.

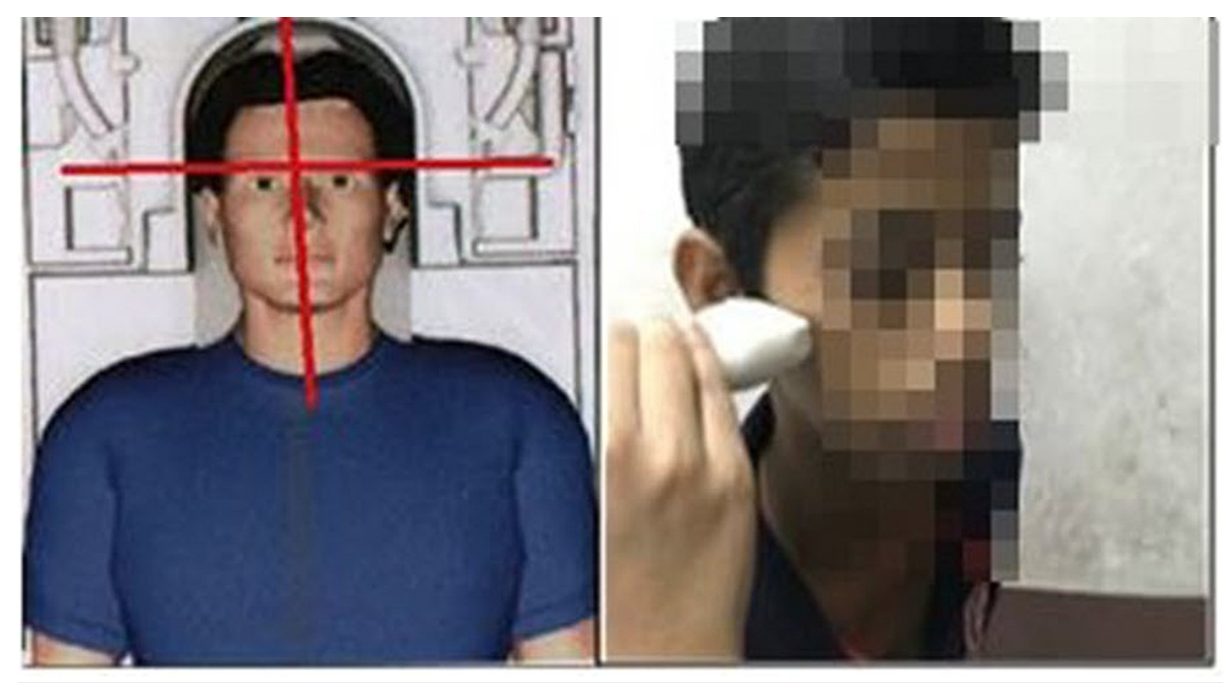

FIGURE 1: Patient position for MRI and ultrasound imaging 


\section{Cureus}

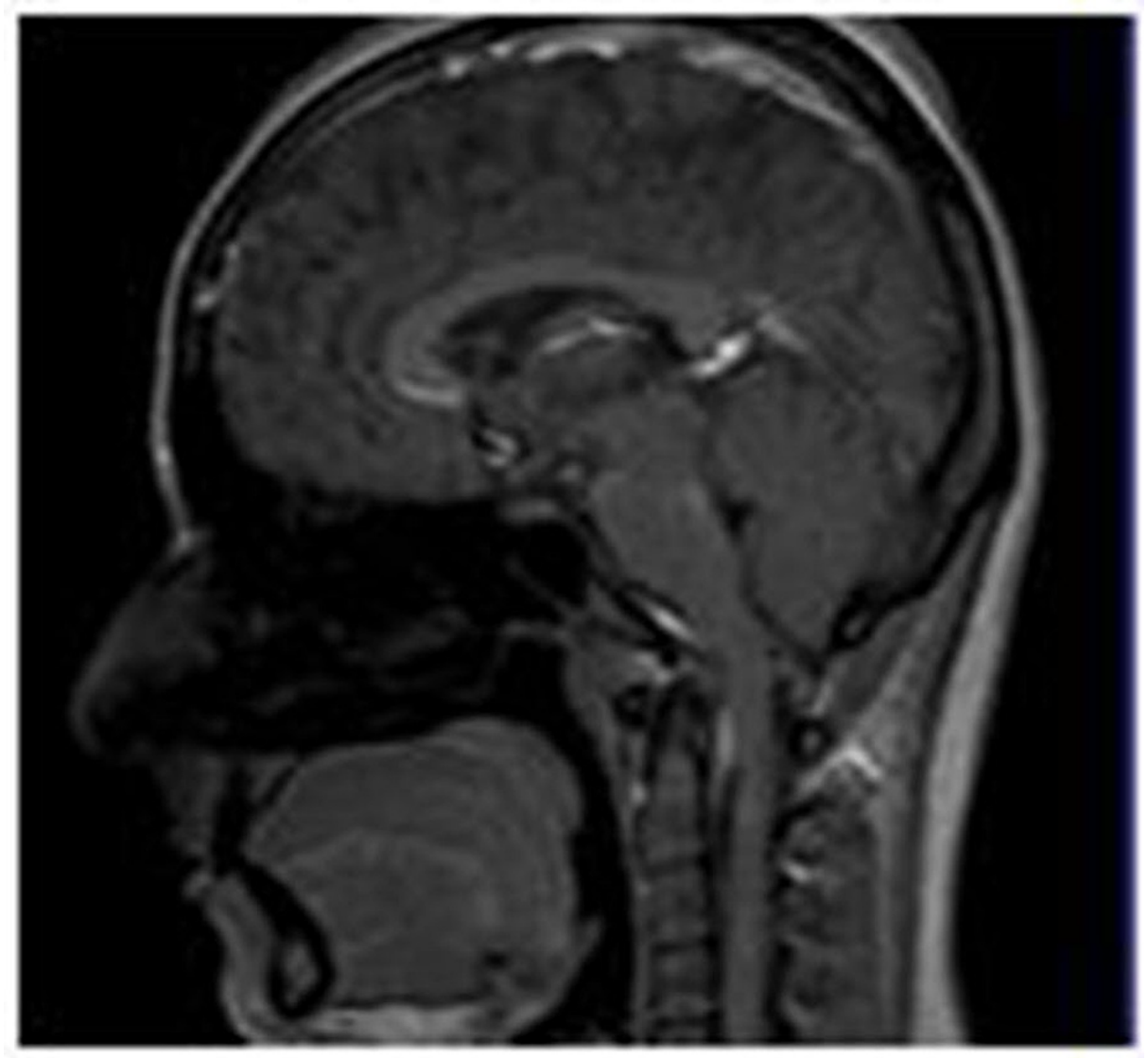

FIGURE 2: Sagittal view of TMJ using the MRI imaging modality

TMJ: temporomandibular joint

A comparative evaluation was done of all the data obtained from the MRI images and ultrasound imaging at $\mathrm{T} 0$ and T1 to appraise any dimensional changes in the thickness of the condylar cartilage. Figures 3-4 demonstrate the dimensional changes of the condylar cartilage, as evaluated by MRI and ultrasound imaging, respectively.

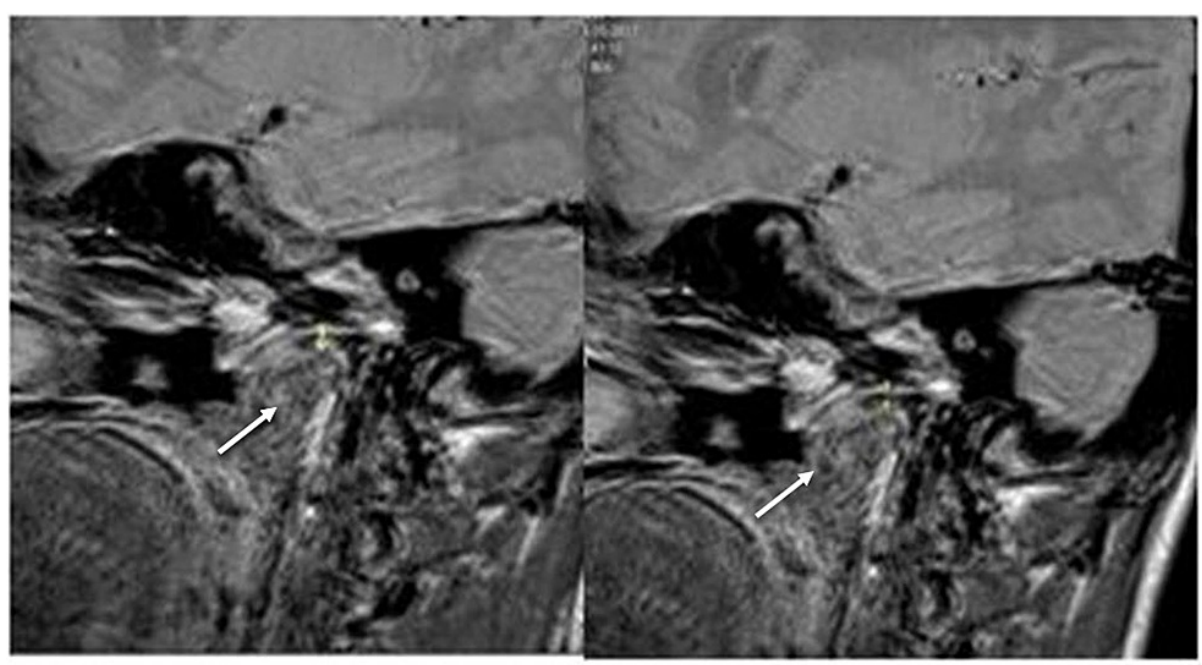

FIGURE 3: MRI images taken at T0 (left) and T1 (right) to demonstrate the dimensional changes of the condylar cartilage after twin-block therapy 


\section{Cureus}

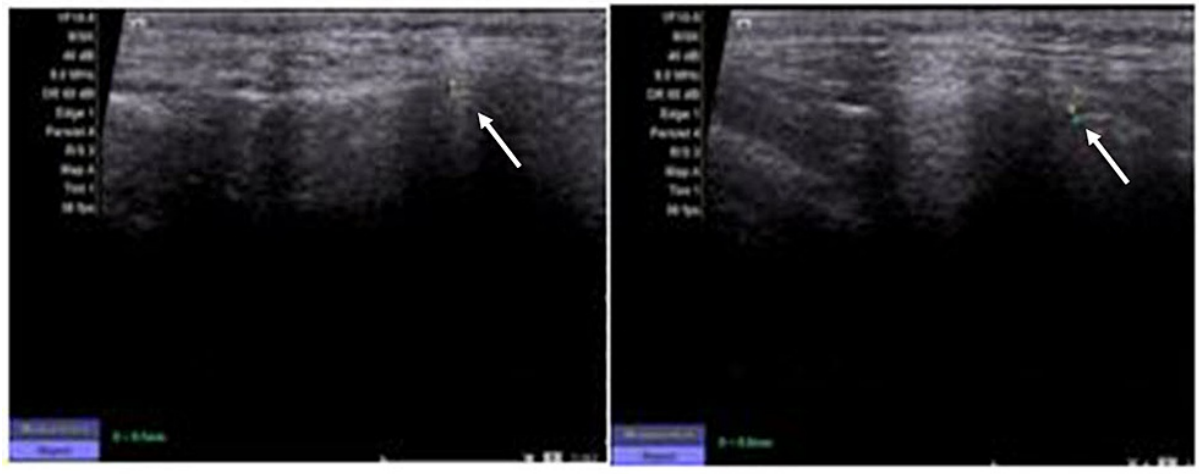

FIGURE 4: Ultrasound images taken at T0 (left) and T1 (right) to demonstrate the dimensional changes of the condylar cartilage after twin-block therapy

Data were analyzed by a between-group comparison using the unpaired t-test and a within-group comparison using the paired t-test.

\section{Results}

There were statistically significant changes in p-value at $\mathrm{T} 0$ and $\mathrm{T} 1$ in the open and closed mouth position, on the left and right sides, as evaluated on MRI. The mean and standard deviations, t-test, and p-value of MRI at T0 and T1 in the open and closed mouth positions on each side are depicted in Table 1.

\begin{tabular}{|c|c|c|c|c|c|c|c|}
\hline Sr. No & Parameter & Mean in $\mathbf{~ m m}$ & $\mathbf{N}$ & Std. Deviation & Mean difference & $t$ value & P-value \\
\hline \multirow{2}{*}{1} & T0 (MRI), open mouth left side & 0.49 & 10 & 0.0876 & -0.28 & -9.635 & $<0.001$ \\
\hline & T1 (MRI) open mouth, left side & 0.77 & 10 & 0.1252 & & & \\
\hline \multirow{2}{*}{2} & T0 (MRI)closed mouth, left side & 0.467 & 9 & 0.0866 & -0.311 & -11.94 & $<0.001$ \\
\hline & T1 (MRI) closed mouth, left side & 0.78 & 9 & 0.044 & & & \\
\hline \multirow{2}{*}{3} & T0 (MRI), open mouth right side & 0.97 & 10 & 1.4174 & 0.16 & 0.356 & 0.73 \\
\hline & T1 (MRI) open mouth, right side & 0.81 & 10 & 0.0994 & & & \\
\hline \multirow{2}{*}{4} & T0 (MRI), closed mouth, right side & 0.52 & 10 & 0.1033 & -0.27 & -12.65 & $<0.001$ \\
\hline & TI (MRI) closed mouth, right side & 0.79 & 10 & 0.0738 & & & \\
\hline
\end{tabular}

TABLE 1: Data obtained from MRIs in the open and closed mouth positions on the left and right sides at $\mathrm{TO}$ and $\mathrm{T} 1$

At T0, the mean thickness noted was 0.49 and 0.48 during opening and closing on the left side and 0.52 in both positions on the right side. At T1, the mean thickness noted was 0.8 and 0.79 during opening and closing on the left side and 0.81 in both positions on the right side. This is graphically represented in Figure 5 . 


\section{Cureus}

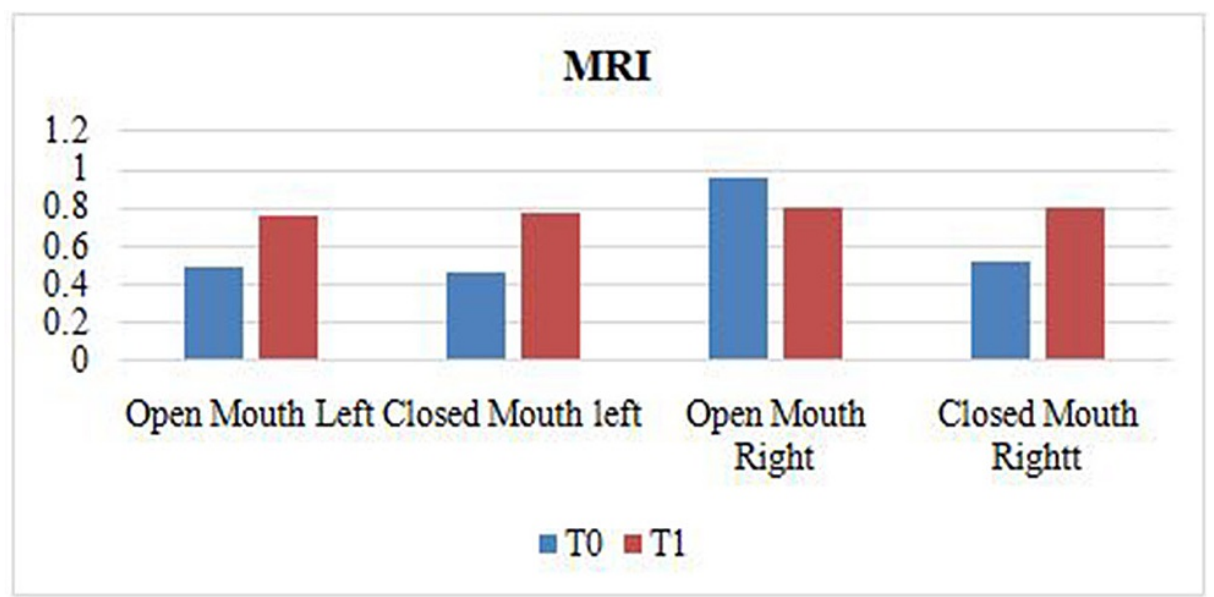

FIGURE 5: Graphical representation of data obtained from MRI images in the open and closed mouth positions on left and right sides at TO and T1

Similarly, ultrasound imaging showed consistent findings that were statistically significant. The mean, standard deviations, t-test, and p-value of ultrasound imaging at $\mathrm{T} 0$ and $\mathrm{T} 1$ in the open and closed mouth positions on each side are depicted in Table 2 .

\begin{tabular}{|c|c|c|c|c|c|c|c|}
\hline Sr no & Parameter & Mean in $\mathrm{mm}$ & $\mathbf{N}$ & Std. Deviation & Mean diff & t-value & P-value \\
\hline 1 & T0 (USG), open mouth left side & 0.49 & 10 & 0.0876 & -0.31 & -13.286 & $<0.001$ \\
\hline 2 & 11 (USG) open mouth, left side & 0.8 & 10 & 0.0816 & & & \\
\hline 3 & T0 (USG)closed mouth, left side & 0.48 & 10 & 0.0919 & -0.31 & -13.286 & $<0.001$ \\
\hline 4 & T1 (USG) closed mouth, left side & 0.79 & 10 & 0.057 & & & \\
\hline 5 & T0 (USG), open mouth right side & 0.52 & 10 & 0.0632 & -0.29 & -12.429 & $<0.001$ \\
\hline 6 & T1 (USG) open mouth, right side & 0.81 & 10 & 0.0994 & & & \\
\hline 7 & T0 (USG), closed mouth, right side & 0.52 & 10 & 0.1033 & -0.29 & -9.222 & $<0.001$ \\
\hline 8 & TI (USG) closed mouth, right side & 0.81 & 10 & 0.0316 & & & \\
\hline
\end{tabular}

TABLE 2: Data obtained from ultrasound imaging in open and closed mouth position on left and right sides at $\mathrm{T} 0$ and $\mathrm{T} 1$

At T0, the mean thickness noted was 0.49 and 0.48 during opening and closing on the left side and 0.52 in both positions on the right side. At TI, the mean thickness noted was 0.8 and 0.79 during opening and closing on the left side and 0.81 in both positions on the right side. This is graphically represented in Figure 6. 


\section{Cureus}

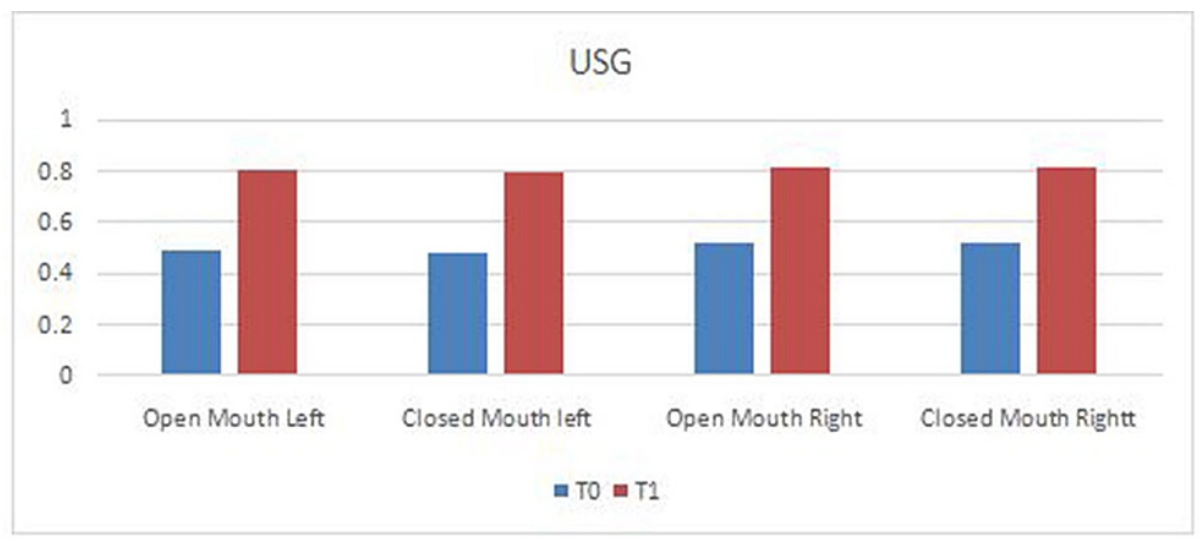

FIGURE 6: Graphical representation of data obtained from ultrasound images in the open and closed mouth positions on the left and right sides at $\mathrm{T} 0$ and $\mathrm{T} 1$

There were no significant differences between the mean thickness of condylar cartilage as interpreted by MRI and ultrasound imaging at T0 and T1. It can be inferred that both imaging modalities are of diagnostic equality, as shown in Figure 7. 

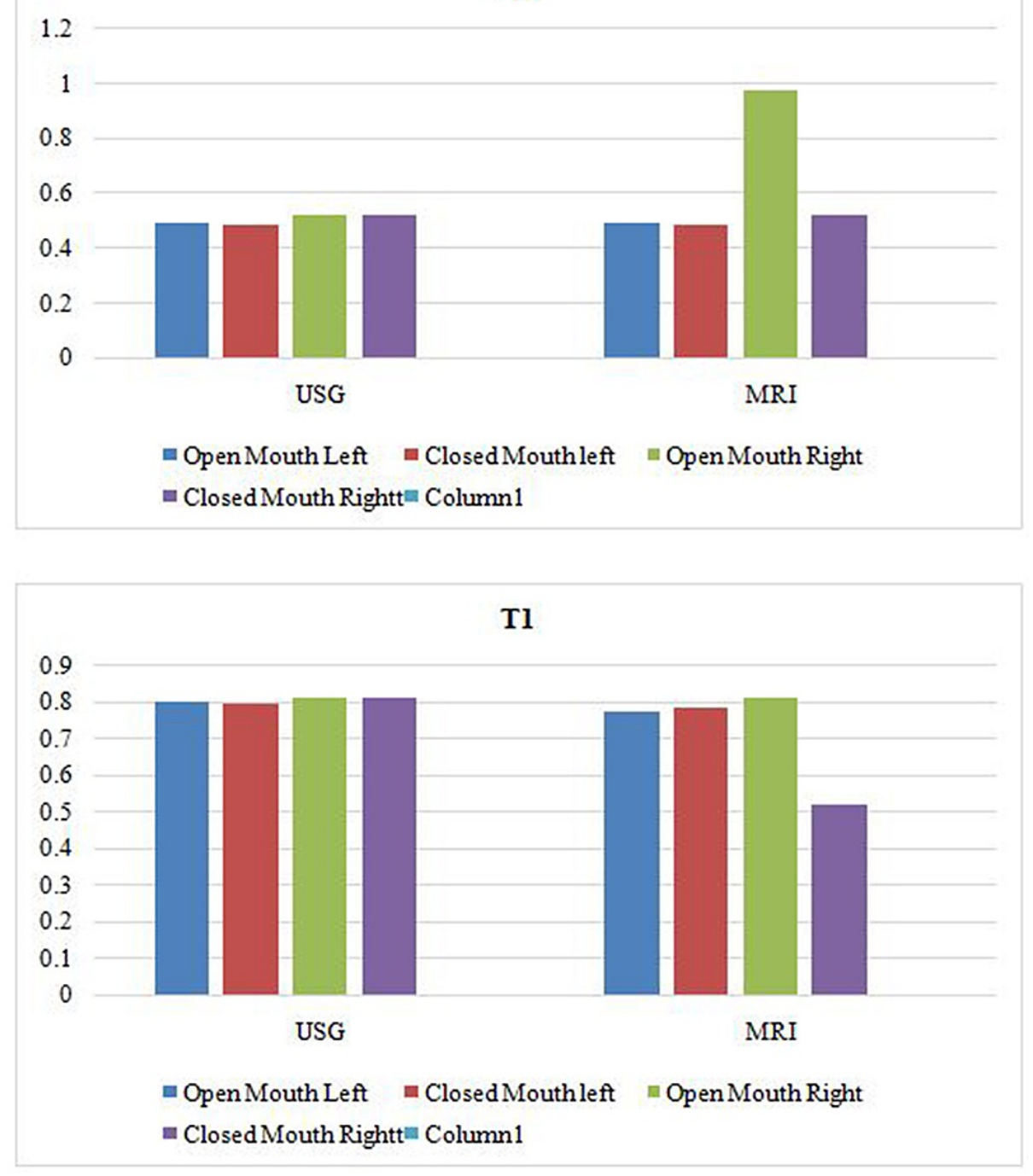

\section{FIGURE 7: Comparative evaluation of data interpreted by MRI and ultrasound imaging at $\mathrm{T} 0$ and $\mathrm{T} 1$}

\section{Discussion}

The alterations within the condyle-glenoid fossa complex and the positional changes of the glenoid fossa in the cranium after removable functional appliance therapy and after the completion of fixed appliance therapy can likewise be noted utilizing MRI. The MRI system is known to be non-intrusive and radiationfree and gives more prevalent difference determination than some other imaging modality [3]. However, it is endowed with several disadvantages such as increased cost and taking longer to procure, thereby minimizing patient comfort. Images are subject to unique artifacts that must be recognized and abated. It is non-compliant for patients with metal implants and foreign bodies, and careful attention to safety measures is necessary to avoid serious injury to patients and staff. Also, it requires special MRI-compatible equipment and stringent adherence to safety protocols. Therefore, we used ultrasound imaging in conjunction with MRI to obtain an insight into the dynamics of the TMJ.

High-resolution USG is an accurate, inexpensive, readily accepted by patients, and non-invasive method for imaging the musculoskeletal system. Articular cartilage can be visualized directly by USG at different peripheral joints, including the knee, elbow, wrist, shoulder, etc. It appears as a homogeneous anechoic band because of the high water content between the bony cortex and the soft tissues delimited by two sharp and hyper-echoic interfaces. The lack of echoes and the sharpness of the synovial space-cartilage and cartilagebone inter-faces are the principal features of articular cartilage in healthy subjects [8-12].

The temporomandibular joint can be visualized using the closed, partially open, and open-mouth positions. MRI is a dependable procedure for evaluating the TMJ when evaluating its normalcy in the closed-and open 
mouth positions [13]. Over the years, imaging modalities like MRI and USG imaging have been readily used for determining the condylar position, disc displacement, glenoid fossa remodeling, and change in mandibular position. However, our study employs MRI and ultrasound imaging for evaluating the condylar cartilage thickness, owing to the effectiveness of the imaging systems and minimized radiation exposure.

Our study designates MRI as the gold standard for examination of the TMJ area for differentiating between soft tissues, evaluating dimensional changes of condylar cartilage. Ultrasound imaging, on the other hand, is a form of mechanical vitality that is transmitted through and into biological tissues as an acoustic pressure wave at frequencies over the farthest point of human hearing. Ultrasound imaging has been used to evaluate the condylar motion, to assess the morphology of masseter muscle detection of condylar involvement in children with juvenile idiopathic arthritis, TMJ disc displacements, and for glenoid fossa remodeling [12]. However, there is a lack of literature evaluating the changes in the thickness of the condylar cartilage after functional appliance therapy using these imaging modalities, necessitating the need for this study.

In 2003, Voudouris et al. attempted to improve on previous studies by combining several methods, including permanent implantation of electromyography (EMG) electrodes, computerized histomorphometry of bone formation, etc. They concluded that the glenoid fossa grows histologically in a downward and backward direction in control of nonhuman primates (similar to growth in humans) [4].

In 1997, Rabie et al. studied osteogenesis in the glenoid fossa in response to mandibular advancement at various time intervals in rats. The remodeling occurs by the expression of endogenous regulatory factors of cells in the mandibular condyle through an endochondral ossification process and intramembranous ossification in the GF [13-14].

In 2014, Santosh et al. evaluated and compared temporomandibular joint changes and the disk-condylefossa relationship following functional appliance therapy using twin-block and Bionator appliances using MRI. This study confirmed that the condyles occupied a more anterior position in the fossa to its pretreatment position while the disk moved more posteriorly in relation to the condyle; these findings are consistent with the findings in our study [1]. Mandibular protrusion leads to an increment in the number of replicating mesenchymal cells in the temporomandibular joint space. Individual variations in the response to growth modification therapy could be a result of the close interrelation between mesenchymal cell numbers and their growth [15-16].

There were moderate to strong correlations between MRI and USG measures of cartilage thickness in the medial femoral condyle in a study done by Schmitz et al. Even though USG may underestimate cartilage thickness relative to MRI in a single sitting, it is actually a useful clinical tool to assess cartilage thickness when serial investigations are performed [7].

In this study, both MRI and ultrasound imaging portrayed consistent findings showing statistically significant changes in p-value at T0 and T1 in the open and closed mouth positions, on the left and right sides. An additional observation noted in this study was that the condylar cartilage thickness of males was more as compared with females of the same age. However, these observations noted were beyond the scope of this study and further research is required to substantiate it. USG is viable and effective in measuring the outer marginal thickness of condylar cartilage, whereas MRI is effective in measuring the entire cartilage thickness. On the contrary, USG is more dynamic to measure thickness in the open and close mouth technique as compared to MRI because of the inherent cumbersome requirements for MRI, whereas USG is a simple chairside and cost-effective procedure. While MRI by its superior resolution of detail, penetration, and depiction of even deeper tissues, is the gold standard for assessing condylar cartilage thickness, the role of USG as a highly valuable diagnostic tool to assess condylar cartilage proliferation, especially when done serially cannot be understated, something which is required to assess the effectiveness of a given mandibular advancement appliance.

\section{Conclusions}

Within the given consensus of this study, we concluded that the condylar cartilage thickness increases significantly after twin-block therapy suggestive of mandibular growth in skeletal Class-II Malocclusion. It can be inferred that both MRI and ultrasound imaging carry equal diagnostic interpretation, as there was no statistically significant difference between the two imaging modalities.

\section{Additional Information}

\section{Disclosures}

Human subjects: Consent was obtained or waived by all participants in this study. Institutional Ethics Committee Dr. DY Patil Dental College and Hospital issued approval DYPDCH/760/2015. Ethics committee approval was granted by the institutional ethics committee Dr. DY Patil Dental College and Hospital as DYPDCH/760/2015. Animal subjects: All authors have confirmed that this study did not involve animal subjects or tissue. Conflicts of interest: In compliance with the ICMJE uniform disclosure form, all authors declare the following: Payment/services info: All authors have declared that no financial support was 
received from any organization for the submitted work. Financial relationships: All authors have declared that they have no financial relationships at present or within the previous three years with any organizations that might have an interest in the submitted work. Other relationships: All authors have declared that there are no other relationships or activities that could appear to have influenced the submitted work.

\section{References}

1. Chavan SJ, Bhad WA, Doshi UH: Comparison of temporomandibular joint changes in twin block and Bionator appliance therapy: a magnetic resonance imaging study. Prog Orthod. 2014, 15:57. 10.1186/s40510-014-0057-6

2. Owtad P, Potres Z, Shen G, Petocz P, Darendeliler MA: A histochemical study on condylar cartilage and glenoid fossa during mandibular advancement. Angle Orthod. 2011, 81:270-6. 10.2319/021710-99.1

3. Wadhawan N, Kumar S, Kharbanda OP, Duggal R, Sharma R: Temporomandibular joint adaptations following two-phase therapy: an MRI study. Orthod Craniofac Res. 2008, 11:235-50. 10.1111/j.16016343.2008.00436.x

4. Voudouris JC, Woodside DG, Altuna G. Kuftinec MM: Condyle-fossa modifications and muscle interactions during Herbst treatment, part 1. New technological methods. Am J Orthod Dentofacial Orthop. 2003, 123:604-13. 10.1016/s0889-5406(03)00149-5

5. Santos RA, Silva CS, Noite FS, Andre A: Ultrasound evaluation of the mandibular condyle cartilage thickness and its influence on the temporomandibular joint and masseter muscle. Eur Soc Radiol. 2018, 2018:C-2384. 10.1594/ecr2018/C-2384

6. Williams TG, Holmes AP, Bowes M, et al.: Measurement and visualisation of focal cartilage thickness change by MRI in a study of knee osteoarthritis using a novel image analysis tool. Br J Radiol. 2010, 83:940-8. 10.1259/bjr/68875123

7. Schmitz RJ, Wang HM, Polprasert DR, Kraft RA, Pietrosimone BG: Evaluation of knee cartilage thickness: a comparison between ultrasound and magnetic resonance imaging methods. Knee. 2017, 24:217-23. 10.1016/j.knee.2016.10.004

8. Oksayan R, Sokucu O, Ucuncu N: Effects of bite-jumping appliances on mandibular advancement in growing rats: a radiographic study. Eur J Dent. 2014, 8:291-5. 10.4103/1305-7456.137624

9. Grassi W, Filippucci E, Farina A: Ultrasonography in osteoarthritis. Semin Arthritis Rheum. 2005, 34:19-23. 10.1016/j.semarthrit.2004.03.007

10. Dauravu LM, Vannala V, Arafath M, Singaraju GS, Cherukuri SA, Mathew A: The assessment of sagittal changes with twin block appliance in patients with decelerating growth phase. J Clin Diagn Res. 2014, 8:ZC81-4. 10.7860/JCDR/2014/10747.5354

11. Reicheneder CA, Proff P, Baumert U, Gedrange T: Growth-related differences in maximum laterotrusion and retrusion between children and adults. Angle Orthod. 2009, 79:265-70. 10.2319/011508-21.1

12. deSenna BR, dos Santos Silva VK, França JP, et al.: Imaging diagnosis of the temporomandibular joint: critical review of indications and new perspectives. Oral Radiol. 2009, 25:86. 10.1007/s11282-009-0025-X

13. Drace JE, Enzmann DR: Defining the normal temporomandibular joint: closed-, partially open-, and openmouth MR imaging of asymptomatic subjects. Radiology. 1990, 177:67-71. 10.1148/radiology.177.1.2399340

14. Braun S, Hicken JS: Ultrasound imaging of condylar motion: a preliminary report. Angle Orthod. 2000, 70:383-6.

15. El-Bialy T, El-Shamy I, Graber TM: Growth modification of the rabbit mandible using therapeutic ultrasound: is it possible to enhance functional appliance results?. Angle Orthod. 2003, 73:631-9.

16. Emshoff R, Bertram S, Rudisch A, Gassner R: The diagnostic value of ultrasonography to determine the temporomandibular joint disk position. Oral Surg Oral Med Oral Pathol Oral Radiol Endod. 1997, 84:688-96. 